MS1-P5 Additives changing the adhesion properties of macromolecules during crystallization process. Jindřich Hašek, Tereza Skálová, Jarmila Dušková, Tomáš Koval', Jan Dohnálek Institute of Macromolecular Chemistry, AS CR, 16206 Praha 6, Czech Republic.

E-mail: hasek@imc.cas.cz

Protein crystallization is in many cases regarded as a random process driven by static thermodynamic laws and practical approach rely on random scanning the whole crystallization space for each protein. Study of intermolecular forces responsible for stability of protein crystals can help much in understanding the process of the crystal growth [1]. Here, we try to explain a special role of additives and salts dynamically changing adhesion between protein molecules during their stacking into the regular lattice. Protein crystal is regarded as a well defined block of highly concentrated solution $(20-70 \%)$, and the 3D long range periodicity for a large part of atoms in the unit cell is dynamically ensured by well defined intermolecular forces. Any additives adhering temporally to protein surface in solution block some surface residues which may be decisive for adhesion and deposition of protein molecule to the surface of growing crystal. Especially, large molecules (e.g. the hydrophilic polymers or the PEG-based detergents [1]) adhering selectively on specific spots on protein surface important for crystalline scaffold play an important role in crystallization because they sterically block the incompatible adhesion modes increasing thus the probability of crystallization and the crystal quality.

We call these additives "Protein Surface Shielding Agents (PSSA)" [2]. Because of their large surface exposed to solvent, the unprotected protein molecules have always many mutual adhesion modes competing in deposition of each protein molecule into the growing crystal. The higher probability of some competitive adhesion mode which is incompatible with the dominant adhesion mode in the same crystal lattice leads to the increasing rate of wrong depositions of single molecules (or rows or blocks of molecules) leading thus to non-diffracting precipitate or purely diffracting crystals. It is evident that a selective blocking the incompatible adhesion modes during crystallization increases diffraction quality of crystals. Playing with the PSSA's blocking different adhesion modes is also used for crystallization of the protein in different space groups, i.e. in different molecular environments. It allows scanning the association potential of proteins and visualizing the conformational changes induced by intermolecular forces. The association potential of PSSA's is lower than the one of protein and thus PSSA's are usually expelled from their position on the growing surface. However, in some cases $(\sim 5$ $\%$ cases) they remain built in well defined positions on the protein surface, so that we know their behaviour very well. Several thousands of examples in the PDB show that the most frequent binding interactions of PEG-based polymers are [1]:

- chelating of several ether oxygens on tips of Lys, Arg, His,

- cation chelated by PEG oxygens binds to carbonyl groups,

- multiple H-bonds in H-bond donor rich grooves,

- combination hydrophobic and $\mathrm{CH} 2-\pi$ interactions namely in the Trp rich clefts of some transporting proteins.

The knowledge of shielding propensities of different PSSA's allows us to increase the crystallization rate and reduce the number of crystallization trials.

[1] Hašek, J. (2006) Z. Kristallogr. 23, 613-619.

[2] Hašek J. et al, (2011) Z. Kristallogr. 28, 475-480.

Keywords: protein crystallization; intermolecular forces; hydrophilic polymers; crystallization additives

\section{MS1-P6 The Norwegian Structural Biology Centre} Ronny Helland, Arne O. Smalĺs, University of Tromsø, Department of Chemistry, Norway

E-mail: ronny.helland@uit.no

The Norwegian Structural Biology Centre (NorStruct) was established as a national laboratory for 3D structural analysis within the functional genomics (FUGE) initiative in Norway. The Centre offers consulting, service, courses and collaboration to the Norwegian research community in structural biology techniques. The lab is equipped with state-of-the-art instrumentation for the full pipeline from protein identification and production, through high throughput crystallization and structure determination to drug discovery related tasks.

Research at NorStruct covers a wide variety of themes, but in-house research projects primarily focus on four topics:

I) Signal transduction - Anti-cancer drug discovery and design

II) DNA intraction and modification

III) Host-pathogen interactions

IV) Bioprospecting, design and development of proteins with medical, biotechnological and commercial potential

Keywords: protein crystallography, bioprospecting, extremeophilic enzymes 